$100 \mathrm{ml}$ aufgefüllt. Die Ansätze wurden 30 Min. bei Raumtemperatur stehengelassen und dann $0,25 \mathrm{~m} /$ der so erhaltenen etwa $0,5 \mathrm{mM}$ Lösungen wie vorstehend angegeben zu $10 \mathrm{ml} 25 \mu \mathrm{M}$ Mercuribenzoatlösung gegeben und der $p$-Mercuribenzoat-Uberschuß nach 30 Min. mit 0,5 mM Cysteinlösung zurücktitriert.

Alkalische Hydrolyse von Benzylpenicillin

Etwa $50 \mu \mathrm{Mol}$ Benzylpenicillin wurden genau eingewogen, in einem $100 \mathrm{~m}$-Meßkolben mit $16,5 \mathrm{ml}$ 1N NaOH versetzt und bei Raumtemperatur einige Stdn. stehengelassen. (Bei einer Hydrolysedauer zwischen 1 und $24 \mathrm{Stdn}$. ergaben die nachfolgenden Bestimmungen mit $p$-Mercuribenzoat annäbernd gleiche Werte.)
Dann wurde mit $33 \mathrm{ml}$ 1N Essigsäure abgepuffert (es stellt sich ein pH-Wert von 4,6 ein) und auf $100 \mathrm{ml}$ aufgefüllt. $0,25 \mathrm{ml}$ der so erhaltenen etwa 0,5 mM Lösungen der Hydrolyseprodukte wurden wie oben angegeben mit $p$-Mercuribenzoat bestimmt.

Wir danken den Farbwerken Hoechst AG für die kostenlose Uberlassung von Penicillinen und Penicillinase, den Farbenfabriken Bayer AG für Penicillin, der Fa. Heyl \& Co. für Penicillin und Penicillamin und der Fa. Schering für die Durchführung der $\mathrm{C}, \mathrm{H}-\mathrm{B}$-stimmung.

Frau Barbara Wrnhoff und Fräulein Brigitte Clemens $\dagger$ danken wir für ihre technische Assistenz.

\title{
Literatur
}

1. Siegmund, P. und F. Körber, Hoppe-Seylers Z. physiol. Chem. 349, 317 (1968). - 2. Boyre, P. D., J. Amer. chem. Soc. 76, 4331 (1954). - 3. Chinard, F. P. und L. Hellermann, Method biochem. Analysis 1, 1 (1954). - 4. BeNESCH, R. und R. E. Benesch, Methods biochem. Analysis 10, 43 (1962). - 5. The Chemistry of Penicillin, S. 960, Hrsg. H. T. Clarke, J. R. Johnson und Sir R. Robinson, Princeton Universtiy Press, Princeton, New Jersey (1949). - 6. Schubert, M. P., J. biol. Chemistry 121, 539 (1937). - 7. Weitzel, G., J. ENGELMANN und A.-M. FretzdorfF, Hoppe-Seylers Z. physiol. Chem. 315, 236 (1959). 8. Ratner, S. und H. T. Clarke, Amer. chem. Soc. 59, 200 (1937).
9. Böhme, H. und H. Wojarn, Deutsches Arzneibuch, 6. Ausgabe, 3. Nachtrag, Kommentar S. 321-333. Wiss. Verlags-Gesellschaft $\mathrm{mbH}$ Stuttgart und Govi-Verlag GmbH Frankfurt/Main (1959). - 10. Perlin, A. S. und C. Brice, Canad. J. Chem. 34, 85 (1956). - 11. Merz, K. W., H. KNIEps und H. LehmanN, Pharmazie 20, 764 (1965). - 12. Armstrong, M. D. und V. DU Vigneaud, J. biol. Chemistry 168, 373 (1947). - 13. Armstrong, M. D. und V. DU Vigneaud, J. biol. Chemistry 173, 749 (1948). - 14. Pollock, M. R., Penicillinase, in The Enzymes, 2. Auf. Bd. 4 S. 269, Hrsg. P. D. Boyer, H. Lardy und K. Myrbäck, Academic Press New York und London (1960).

\section{Lokalisation und Charakterisierung der Lipase- bzw. Tributyrinase-Aktivitäten im perirenalen Fettgewebe vom Schwein}

\author{
Von U. H. KLEmens \\ Aus dem Pbysiologisch-Chemischen Institut der Freien Universität Berlin. (Direktor: Prof. Dr. Dr. E. Scbütte)
}

(Eingegangen am 6. April 1968)

Herrn Prof. Dr. Dr. Ernst Scbütte zum 60. Geburtstag gewidmet

Im perirenalen Fettgewebe vom Schwein werden zwei Lipasen und eine Tributyrin spaltende Esterase nachgewiesen. Nach der Enzymcharakteristik handelt es sich um eine Lipoproteid-Lipase, die dem Klärfaktor des Blutes (1, 2) bzw. der pH-8,5-Gewebslipase (3) ähnelt, sowie um eine Lipase, die viele Gemeinsamkeiten mit der Adrenalin-empfindlichen Lipase $(3,4)$ hat. Die Lipoproteid-Lipase und Tributyrinase sind überwiegend im Uberstand des Ultrazentrifugates $(120000 \mathrm{~g}, 2$ Stdn.) lokalisiert. Das dritte beschriebene Enzym erscheint im Sediment.

Wir bestimmten die Substratspezifität, die $\mathbf{p H}$-Optima, die $\mathrm{K}_{\mathbf{m}}$-Werte, die Stöchiometrie der Lipolyseprodukte, untersuchten die Stabilität der Enzyme unter verschiedenen Bedingungen, die Beeinflussung der Enzymaktivitäten durch bestimmte Inhibitoren bzw. Aktivatoren, und versuchten, die Enzyme in der Zwischenphase von hydrophoben und hydrophilen Medien anzureichern.

Two lipases and a tributyrin-cleaving esterase were demonstrated in the perirenal fat tissue of the pig. One of the lipases had the properties of a lipoprotein lipase, similar to the clearing factor of blood $(1,2)$, or the $\mathrm{pH} 8.5$ - tissue lipase (3), and the other lipase had many properties in common with the adrenaline-sensitive lipase $(3,4)$. The lipoprotein lipase and the tributyrinase are localised predominantly in the supernatant of the ultracentifugate $(120,000 \mathrm{~g}, 2 \mathrm{hr}$.). The third enzyme was found in the sediment.

The substrate specificity, $\mathrm{pH}$-optima and $\mathrm{K}_{\mathrm{m}}$-values of the enzymes, and the stoichiometry of the lipolysis products were determined. The stability of the enzymes under different conditions and the effect of inhibitors and activators on enzyme activity were studied; and it was attempted to concentrate the enzyme in the intermediate phase of hydrophobic and hydrophilic media.

In verschiedenen Geweben, z. B. im epididymalen Fettgewebe $(3,4,5)$, im Herzmuskel der Ratte $(2,4,6)$, und in der menschlichen Lunge (7) wurden Lipasen nachgewiesen und charakterisiert. Dabei konnten $z$ wiei Gewebslipasen differenziert werden:

1. Eine Lipoproteid-Lipase (2) bzw. pH-8,5-Lipase (3), die wahrscheinlich identisch sind. Beide haben viele Gemeinsamkeiten mit dem Klärfaktor des Blutplasmas
(1). Die Lipaseaktivitäten können durch Heparin beeinflußt werden. Man findet einen Anstieg der Lipaseaktivität im Puffermedium einer Fettgewebsinkubation, wenn zum Inkubationsgemisch Heparin hinzugegeben wird (3).

2. Eine Adrenalin-empfindliche Lipase $(3,4)$ die - im Gegensatz zur Lipoproteid-Lipase - im Fettgewebe von. mageren Tieren eine größere Aktivität dls bei 
fetten Tieren bat $(3,4,8,9)$. Wenn Fettgewebe vor dem Homogenisieren in einer Adrenalin- oder ACTHLösung inkubiert wurde, so erscheint im Homogenat mehr lipolytische Aktivität (10).

Wir stellten uns die Aufgabe, die Lipasen bzw. die Tributyrinase des perirenalen Fettgewebes vom Schwein zu klassifizieren. Im Zusammenhang mit der Heparininduzierten Abgabe der Lipoproteid-Lipase aus dem Gewebe in das Inkubationsmedium interessierte die Lokalisation der gefundenen Lipasen in der Zelle. Wir untersuchten deshalb ihre quantitative Verteilung auf verschiedene, durch Zentrifugieren gewonnene Zellfraktionen.

\section{Methodik}

\section{Aufarbeitung des Fettgenvebes}

Unmittelbar nach dem Schlachten der Tiere wurde das Fettgewebe entnommen und sofort in flüssigen Stickstoff eingebracht. Das gefrorene Gewebe wurde gemörsert. Das Fettgewebspulver ist bei $-30^{\circ}$ mehrere Wochen haltbar. Wegen der Instabilität der Lipasen gegenüber Wärme wurde bei der weiteren Aufarbeitung auf eine konstante Temperatur von $2-4^{\circ}$ geachtet. $5 \mathrm{~g}$ Fettgewebspulver wurden je nach Fragestellung in $20 \mathrm{ml} 0,05 \mathrm{M}$

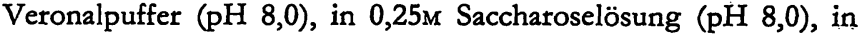
$0,05 \mathrm{M}$ Phosphatpuffer (verschiedener pH-Werte), oder in bidest. Wasser suspendiert und mit dem Ultraturrax homogenisiert. Beim anschließenden Zentrifugieren in der Kühlzentrifuge $(12100 \mathrm{~g}$, 15 Min.) bildeten sich 3 Schichten, ein Sediment, ein Fettüberstand und eine wäßrige $Z$ wischenschicht. Die Zwischenschicht wurde, wenn bei den einzelnen Versuchen nicht anders angegeben, in der Ultrazentrifuge (Spinco L 50) bei $120000 \mathrm{~g} 2$ Stdn. zentrifugiert. Das Sediment wurde in einer der oben genannten Pufferlösungen bzw. Wasser suspendiert (Enzymlösung). Im Abschnitt 1 der Ergebnisse sind die Lipaseaktivitäten aller 5 aufgeführten Fraktionen bilanzmäßig erfaßt. Bei der Charakterisierung der Lipasen wurden die Enzymaktivitäten der beiden Ultrazentrifugenfraktionen unter verschiedenen Bedingungen ermittelt.

\section{Inkubationsansatz}

Die Enzymlösungen wurden so verdünnt, daß $1 \mathrm{~m} /$ aus den verschiedenen Substraten in der Minute etwa 2-3 $\mu \mathrm{g}$ Glycerin freisetzte. Parallel wurde ein Leerwert (Inaktivierung der Enzyme im siedenden Wasserbad, $10 \mathrm{Min}$.) angesetzt. 2 Vol. Enzymlösung bzw. inaktivierte Enzymlösung wurden mit 1 Vol. Substrat unter Schütteln im Wasserbad bei $37^{\circ}$ inkubiert. Der pHWert des Inkubationsgemisches wird bei den einzelnen Versuchen angegeben. Die Reaktion wurde nach $15 \mathrm{Min}$. durch Einbringen in ein siedendes Wasserbad (10 Min.) gestoppt. Die ausgefallenen Proteine wurden in der Kühlzentrifuge bei $49500 \mathrm{~g}$ (10 Min.) sedimentiert. Im Überstand wurden Glycerin und freie Fettsäuren quantitativ bestimmt.

Die Substrate wurden mit demselben Puffer wie die Enzymlösung angesetzt.

Verwendet wurden: a) 2proz. Tributyrinsuspension, b) 5proz. Trioleinsuspension, c) 5 proz. Trioleinsuspension mit Albumin in einer Konzentration von $2,5 \%$, d) 5 proz. aktivierte Trioleinsuspension (1). (0,5 g reines Triolein wurde mit $9,5 \mathrm{ml}$ Serum$0,05 \mathrm{M}$ Veronalpuffer ( $\mathrm{pH} 8,0$, Serum-Puffer-Verhältnis $1: 1$ ) bei $37^{\circ} 2 \mathrm{Stdn}$. intensiv durchmischt. Dabei sollen Lipoproteide entstehen (1).) Die einzelnen Suspensionen wurden jeden Tag frisch angesetzt.

\section{Bestimmung von Glycerin und freien Fettsäuren}

a) Glycerin wurde enzymatisch-optisch mit der Biochemica Test Combination (kombinierte Anwendung von Glycerokinase, Pyruvatkinase und Lactatdehydrogenase) der Firma Boehringer u. Soehne Mannheim, bestimnt. b) Olsäure wurde titrimetrisch mit $0,02 \mathrm{~N} \mathrm{NaOH}$ nach der Extraktion in ein organisches Lösungsmittel bestimmt (11). Bei der enzymatischen Hydrolyse von proteinfreier Triolein- bzw. Tributyrin-Suspension verwendeten wir auch eine Methode, bei der die freiwerdenden Säureäquivalente durch automatische Titration mit $0,02 \mathrm{~N} \mathrm{NaOH}$ in einer $\mathrm{pH}-$-Stat-Anordnung (Radiometer Kopenhagen) bestimmt wurden. Voraussetzung ist, $\mathrm{da} \beta$ im Inkubationsgemisch kein Puffer vorhanden ist.

\section{Spezifische Aktivität}

Sie wird definiert als die Menge Glycerin oder Fettsäure (in $\mu \mathrm{Mol}$ ), die von einem mg Protein der Enzymlösung bei $37^{\circ}$ pro Minute freigesetzt wird. Wir bestimmten die Proteine nach Kjeldahl, mit der Biuret-Methode bzw. dem Folin-Reagens (12).

Zwischenphasen-Anreicherung der Enzyme in Anlebnung an BASKYS (13) Es wurde untersucht, ob sich die Enzyme in der Zwischenphase $z$ wischen organischen und wäßrigen Lösungsmitteln anreichern lassen. Wir beschreiben die Methode für Diäthyläther als organisches Lösungsmittel.

1 Vol. des Ultrazentrifugates $(120000 \mathrm{~g}, 2 \mathrm{Stdn}$.) wurde mit 1 Vol. peroxidfreiem, vorgekühltem Äther bei $2^{\circ}$ vorsichtig 10 Min. lang geschüttelt. Beim Zentrifugieren in der Kühlzentrifuge $(5000 \mathrm{~g}, 10 \mathrm{Min}$.) bildete sich $\mathrm{zwischen}$ organischer und wäßriger Phase eine membranartige $Z$ wischenschicht. Die organische und wäßrige Phase wurden sorgfältig abpipettiert. Der zurückgebliebene Äther wurde aus der Z̈wischenschicht durch Einleiten von Stickstoff entfernt. (Beim Verwenden anderer organischer Lösungsmittcl entfällt das Einleiten des Stickstoffes.) Die Zwischenschicht wurde in $0,05 \mathrm{M}$ Veronalpuffer ( $\mathrm{pH} 8,0$ ) suspendiert. In dieser Suspension und in der wäßrigen Schicht wurden die Enzymaktivitäten bestimmt.

\section{Ergebnisse}

\section{Fraktionierung der Enzymaktivitäten}

Die Gesamtaktivität des Fettgewebshomogenates $(=100 \%)$ verteilt sich nach dem Zentrifugieren $(12100 \mathrm{~g})$ wie folgt auf 3 .Schichten: Im Fettüberstand erscheinen $4-6 \%$, in der wäßrigen Zwischenschicht 85-90\% und im Sediment $10-12 \%$ der Ausgangsaktivität. Die Verteilung der Enzymaktivitäten ist unabhängig davon, ob als Substrat eine Tributyrin-, Triolein- oder aktivierte Triolein-Suspension verwendet wurde.

Die wäßrige Zwischenschicht, die praktisch die gesamte Enzymaktivität enthält, wurde mit der Ultrazentrifuge weiter fraktioniert. Die Vetteilung der Enzymaktivitäten auf Überstand und Sediment ist in der Tabelle 1 zusammengestellt. Man ersieht aus Tabelle 2,

Tab. 1

Prozentuale Verteilung der Enzymaktivitäten der wäßrigen Zwischenschicht $(12100 \mathrm{~g})$ auf Uberstand und Sediment bei verschieden langer Zentrifugation bzw. unterschiedichen g-Zahlen. Die Verteilung war identisch, wenn die Zwischenschicht $0,25 \mathrm{M}$ Saccharose ( $\mathrm{pH} 8,0$ ) oder $0,05 \mathrm{M}$ Veronalpuffer (pH 8,0) enthielt. Ausgangsaktivität: $100 \%$, pH-Wert des Inkubationsgesmisches: $\mathrm{pH} 8,0$

\begin{tabular}{lcccc}
\hline Substrat & {$[g]$} & $\begin{array}{c}\text { Zentrifuga- } \\
\text { tion } \\
\text { [Stdn.] }\end{array}$ & \multicolumn{2}{c}{$\begin{array}{c}\text { Enzymaktivitäten } \\
{[\%]}\end{array}$} \\
& Uberstand & Sediment \\
\hline aktiviertes & & & & \\
Triolein & 71000 & 1 & 71 & 27 \\
& 120000 & 1 & 70 & 32 \\
& 190000 & 1 & 62 & 40 \\
Triolein & 120000 & 6 & 64 & 39 \\
Triolein + & 120000 & 2 & 68 & 34 \\
Albumin & 120000 & 6 & 40 & 59 \\
Tributyrin & 120000 & 2 & 46 & 54 \\
\hline
\end{tabular}


Tab. 2

Zusammenstellung der spezifischen Enzymaktivitäten in Uberstand und Sediment des Ultrazentrifugates $(120000 \mathrm{~g}, 2$ Stdn.). Die Aktivitäten sind in $\mu$ Mol Glycerin angegeben, die von $1 \mathrm{~g}$ Protein der Entäten sind in $\mu \mathrm{Mol}$ Glycerin angegeben, die von $1 \mathrm{~g}$ Protein der En-
zymlösungen freigesetzt wurden. (Enzymlösungen in $0,05 \mathrm{M}$ Veronalpuffer, pH 8,0)

\begin{tabular}{lcc}
\hline Substrat & $\begin{array}{c}\text { Spezifische Aktivität } \\
{\left[\mu \text { Mol/Min: } \cdot \mathbf{g}, \mathbf{3 7}^{\circ}\right]} \\
\text { Uberstand Sediment }\end{array}$ \\
\hline Triolein & 6,1 & 26,7 \\
Triolein + Albumin & 6,8 & 34,2 \\
aktiviertes Triolein & 20,3 & 36,6 \\
Tributyrin & 23,8 & 21,2 \\
\hline
\end{tabular}

daß $1 \mathrm{~g}$ Protein im Überstand etwa die 3-4fache Menge Glycerin freisetzt, wenn als Substrat an Stelle von Triolein aktiviertes Triolein verwendet wurde. Die Zugabe von Albumin steigert dagegen die TrioleinHydrolyse nur unwesentlich. Wenn aber die Hydrolysedauer von 15 auf 60 Min. verlängert wurde, stieg die Hydrolyserate des Trioleins durch den Zusatz von Albumin um etwa 50-60\% an. Dieser Effekt kann durch Hemmung der Lipolyse durch ihre Endprodukte erklärt werden. Während nach $15 \mathrm{Min}$. die freigesetzte Fettsäuremenge noch nicht hemmt, liegt sie nach 60 Min. im Bereich der Hemmkonzentration (s. Abschnitt 3). Durch Bindung der Fettsäuren an Albumin (14) und damit ihre Entfernung aus dem Reaktionsgleichgewicht kann die gehemmte Lipolyse wieder aktiviert werden.

Im Gegensatz zu den Befunden im Überstand steigt die Lipaseaktivität des Sedimentes bereits bei einer Hydrolysedauer von $15 \mathrm{Min}$. um etwa $30-40 \%$ an, wenn an Stelle von proteinfreiem Triolein - Albumin und Triolein eingesetzt wurden. Um denselben Betrag wird auch die Lipolyse gesteigert, wenn aktiviertes Triolein als Substrat verwendet wurde.

\section{Substratspezifität}

Die höhere Aktivität der Lipase des Überstandes gegenüber aktiviertem Triolein deutet darauf hin, daß es sich um eine Lipoproteid-Lipase handelt. Aktiviertes Triolein enthält Lipoproteide (1).

- Die Aktivität der Sediment-Lipase ist jedoch gegenüber einer aktivierten Trioleinsuspension nicht wesentlich größer als gegen Triolein + Albumin. Die SedimentLipase ist also keine Lipoproteid-Lipase.

\section{Lipolyseprodukte}

Die Lipaseaktivität nimmt ab, wenn die Hydrolyse länger als $20 \mathrm{Min}$. dauert. (Abb. 1). Sie kannn durch Zusatz von Ca-Acetat bzw. Albumin zum Inkubationsgemisch wieder aktiviert werden. Beide Substanzen binden Fettsäuren und können dadurch die Endprodukthemmung der Lipolyse aufheben.

Wir untersuchten in diesem Zusammenhang die Hemmung der Hydrolyse von aktiviertem Triolein durch steigende Ölsäurekonzentrationen im Inkubationsgemisch. Bei einer Konzentration von $8,9 \mu \mathrm{Mol} / \mathrm{ml}$ wurde die Lipase-Aktivität um 40-50\% gehemmt. Um die Verfälschung der. Ergebnisse durch die Endpro-

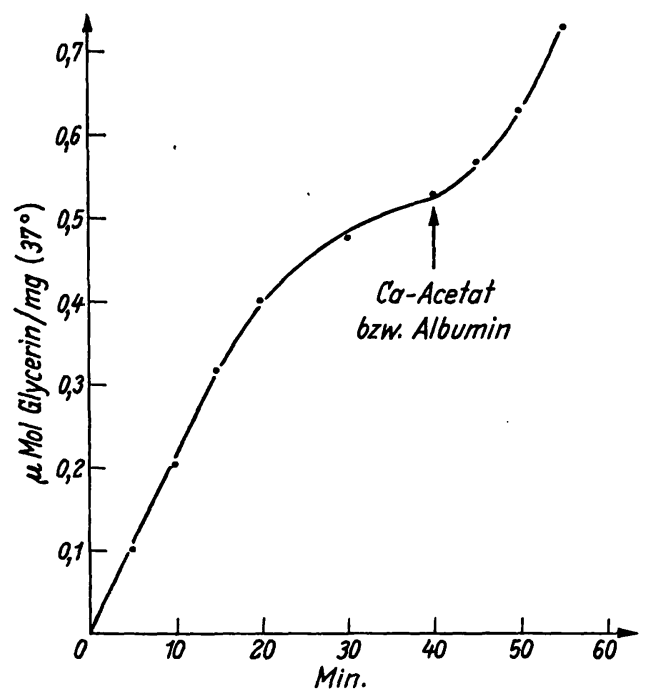

Abb. 1

Abhängigkeit der Lipaseaktivität von der Hydrolysedauer Ordinate: Freigesetzte Menge Glycerin ( $\mu \mathrm{Mol}$ ) pro $\mathrm{mg}$ Protein der Enzymlösung. Abszisse: Hydrolysedauer (Min.). Enzymlösung: Uberstand (in $0,05 \mathrm{M}$ Veronalpuffer). Substrat: aktiviertes Triolein. Nach 40 Min. Zugabe von Ca-Acetat bzw. Albumin zum Inkubationsansatz bis zu einer Konzentration von $0,03 \mathrm{M}$ bzw. 2,5\%

dukthemmung auszuschließen, wählten wir eine kurze Hydrolysezeit von 15 Min., die mit der Enzymkonzentration in der Enzymlösung abgestimmt ist.

Weiter untersuchten wir das stöchiometrische Verhältnis der Lipolyse-Produkte. Die Lipoproteid-Lipase bzw. die Sediment-Lipase setzen aus einer proteinfreien bzw. aktivierten Triolein-Suspension pro Mol Glycerin etwa 9-10 Mol Fettsäuren frei. Dies bedeutet, daß nur etwa 30\% der Ölsäure aus vollständig hydrolysiertem Triolein stammen kann. Der Rest resultiert aus der Teilhydrolyse zu Di- bzw. Mono-Oleat.

\section{4. $\mathrm{pH}$-Optimum}

Der optimale Bereich der Lipaseaktivität des Sedimentes liegt, unabhängig von der Art des Substrates, zwischen $\mathrm{pH}$ 6,5-6, 8. Im Überstand dagegen ist eine Abhängigkeit des pH-Optimums von der Art des Substrates zu erkennen (Abb. 2). Bei der enzymatischen Hydrolyse von aktiviertem Triolein ist die spezifische Aktivität bei $\mathrm{pH} 8,0-8,2$ am größten. Verwendet man aber proteinfreies Triolein als Substrat, dann zeigt sich ein $z$ weites pH-Optimum bei $\mathrm{pH}$ 6,5-6,8. Die Erklärung dafür ist, daß im Überstand des Ultrazentrifugates neben der Lipoproteid-Lipase auch noch eine geringe Menge Sediment-Lipase vorkommt. Deshalb kommt es zur Ausbildung eines zweiten pH-Optimums (Substrat: Triolein) in einem $\mathrm{pH}-$ Bereich, der sich mit dem pH-Optimum der Sediment-Lipase deckt. Dieses zweite pH-Optimum tritt bei der Hydrolyse von aktiviertem Triolein deshalb nicht hervor, weil es durch die große Aktivität der Lipoproteid-Lipase gegenüber diesem Substrat nivelliert wird. Es ist dagegen deutlich ausgeprägt, wenn in Abweichung zu den StandardBedingungen nur 1 Stunde bzw. bei $71000 \mathrm{~g}$ zentrifugiert wurde.

Die Frage der Substratspezifität der LipoproteidLipase (Abschnitt 2) kann demnach dahingehend 


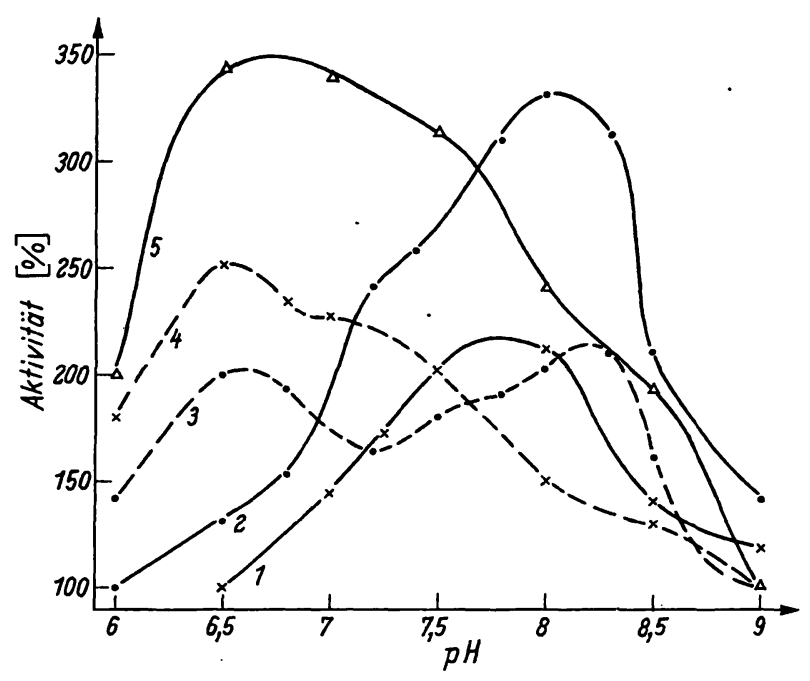

Abb. 2

pH-Optima der Enzymaktivitäten der Ultrazentrifugenfraktionen. Als Enzymlösungen (in $0,05 \mathrm{M}$ Phosphatpuffer) bzw. als Substrate wurden eingesetzt: Versuchsreihe (1) Uberstand, Tributyrin

(2) Uberstand, aktiviertes Triolein, (3) Uberstand, Triolein,

(4) Sediment, aktiviertes Triolein, (5) Sediment, Triolein.

Ordinate: Spezifische Aktivität in \% der kleinsten Aktivität derselben Versuchsreihe. Abszisse: pH-Werte der Inkubationsansätze. Die Inkubationsbedingungen wurden in der Methodik beschrieben

ergänzt werden, daß das Enzym neben aktiviertem Triolein (optimales Substrat) mit geringerer Aktivität auch proteinfreies Triolein hydrolysieren kann.

\section{Stabilität der Enzyme}

a) bei Lagerung:

Fettgewebspulver, sowie Überstand und Sediment (nach Gefriertrocknung), können ohne Aktivitätsverlust bei $-30^{\circ}$ mehrere Wochen gelagert werden. Dagegen sind die Lipasen in wäßrigen Medien instabil. Die Aktivitäten der Lipoproteid-Lipase bzw. der Sediment-Lipase nehmen nach 24 Stdn. bei $4^{\circ}$ um etwa $40 \%$ bzw. 30\% ab. Die Lipasen können gegen dest. Wasser etwa 6-8 Stdn. bei $4^{\circ}$ ohne wesentlichen Aktivitätsverlust dialysiert werden.

b) gegenüber Hitze:

Aus der Tabelle 3 ergibt sich die Wärmeempfindlichkeit der einzelnen Enzyme: Lipoproteid-Lipase > Sediment-

Tab. 3

Wärmeempfindlichkeit der Enzymie. Angegeben sind die spezifischen Aktivitäten in \% der Ausgangsaktivitäten (ohne Erwärmen). Die Enzyme wurden vor der Aktivitätsbestimmung ( $\mathrm{pH} \mathrm{8,0)}$ in Wasser bädern mit verschiedenen Temperaturen $10 \mathrm{Min}$. inkubiert

\begin{tabular}{lrrrrrr}
\hline & $40^{\circ}$ & $45^{\circ}$ & $48^{\circ}$ & $50^{\circ}$ & $60^{\circ}$ & $70^{\circ}$ \\
\hline Lipoproteid-Lipase & 88 & 52 & 30 & 12 & 4 & 0 \\
Sediment-Lipase & 95 & 75 & 50 & 35 & 5 & 0 \\
Tributyrinase & 100 & 100 & 95 & 90 & 18 & 5 \\
\hline
\end{tabular}

Lipase $>$ Tributyrinase. Die Inaktivierung der Lipasen durch Wärme $\left(50^{\circ}, 10\right.$ Min.) kann durch Cystein in einer Konzentration von $0,5 \mathrm{~mm}$ weder verhindert noch aufgehoben werden.

\section{Michaelis-Menten-Konstanten}

In beiden Ultrazentrifugenfraktionen wurde die Beziehung $z$ wischen Enzymaktivität und Substratkonzentration untersucht (Inkubationsmedium: 0,05M Veronalpuffer, $\mathrm{pH} 8,0$ ).

Wir ermittelten folgende $\mathrm{K}_{\mathrm{m}}$-Werte:

a) Lipoproteid-Lipase (Substrat: aktiviertes Triolein): $4,5 \mathrm{~mm}$

b) Sediment-Lipase (Substrat:Triolein): 1,9 mM

c) Tributyrinase (Substrat: Tributyrin): 2,7 mM.

\section{Inhibitoren}

In der Tabelle 4 sind die Etgebnisse zusammengestellt. Charakteristisch für die Lipopröteid-Lipașe ist die

Tab. 4

Zusammenstellung der Hemmsubstanzen. Dargestellt sind die spezifischen Aktivitäten der Enzyme in \% der Ausgangsaktivität (ohne Inhibitor). Die Enzymaktivitäten wurden bei pH 8,0 bestimmt. Substrat: Tributyrin bzw. aktiviertes Triolein

\begin{tabular}{llccc}
\hline Inhibitor & $\begin{array}{c}\text { Konzentra- } \\
\text { tion }\end{array}$ & $\begin{array}{c}\text { Lipoproteid- } \\
\text { Lipase }\end{array}$ & $\begin{array}{c}\text { Sediment- } \\
\text { Lipase }\end{array}$ & $\begin{array}{c}\text { Tributyri- } \\
\text { nase }\end{array}$ \\
\hline Protamin & $0,33 \mathrm{mg} / \mathrm{ml}$ & 45 & 80 & 100 \\
& $0,66 \mathrm{mg} / \mathrm{ml}$ & 30 & 55 & 97 \\
$\mathrm{NaCl}$ & $1,00 \mathrm{M}$ & 20 & 70 & 92 \\
$\mathrm{NaF}$ & $0,22 \mathrm{M}$ & 60 & 40 & 20 \\
& $0,44 \mathrm{M}$ & 55 & 35 & 15 \\
Pyrophosphat & $0,0125 \mathrm{M}$ & 18 & 40 & 55 \\
Phosphat & $0,0125 \mathrm{M}$ & 100 & 100 & 100 \\
& $0,600 \mathrm{M}$ & 75 & 85 & 100 \\
pCMB & $0,25 \mathrm{mM}$ & 15 & 50 & 100 \\
pCMB & $0,25 \mathrm{mM}$ & & & \\
Cystein & $0,25 \mathrm{mM}$ & 95 & 105 & 105 \\
peroxid-haltiger & & 30 & 60 & 80 \\
Äther & & 84 & 91 & 75 \\
EDTA & $0,004 \mathrm{M}$ & & & \\
\hline
\end{tabular}

1) $p \mathrm{CMB}=p$-Chlormercuribenzoat

starke Hemmung durch Protamin, $\mathrm{NaCl}$ und Pyrophosphat. Die Hemmung durch Protamin kann durch Zusatz von äquivalenten Mengen Heparin zum Inkubationsgemisch rückgängig gemacht werden. Auffallend ist die stärkere Hemmung der LipoproteidLipase im Vergleich zur Sediment-Lipase durch $p$ Chlormercuribenzoat und peroxidhaltigen Äther. NaF hemmt dagegen die Sediment-Lipase stärker und besonders stark die Tributyrinase. Alle 3 Enzyme werden in gleicher Weise durch proteolytische Fermente inaktiviert.

\section{Aktivatoren}

Die Steigerung der Lipase-Aktivität durch Ca-Acetat bzzw. Albumin wurde in den Abschnitten 2 und 3 beschrieben. Die Hydrolyse des Tributyrin wird durch Ca-Acetat nicht beeinflußt. Heparin aktiviert die Lipoproteid-Lipase nicht $(1-50 \mathrm{E} / \mathrm{m} \mathrm{l})$.

Cystein $(0,2 \mathrm{~mm})$ vermag die Enzymaktivitäten nicht zu steigern, wenn die Enzymlösungen frisch hergestellt waren. Dagegen nahm die Aktivität beider Enzyme um etwa $30-40 \%$ zu, wenn die Enzymlösungen 5 bis 6 Tage lang eingefroren waren. Cystein kann also die "gealterten“ Lipasen reaktivieren. 


\section{Zwischenphasenanreicherung in Anlehnung an} BASKYS (13)

Es wurde versucht, die Enzyme des Úberstandes $(120000 \mathrm{~g}, 2$ Stdn.) $z$ wischen organischer und wäßriger Phase anzureichern. Als organische Lösungsmittel verwendeten wir Diäthyläther, Hexan, Butanol, Amylalkohol. Die Enzymlösung (in 0,05 M Veronalpuffer, $\mathrm{pH} 8,0$ ) bildete die wäßrige Phase. Es zeigte sich, daß nur Äther als organische Phase verwendet werden kann (peroxidfreil). Etwa $70-80 \%$ der LipoproteidLipase-Aktivität der Enzymlösung wird in der Zwischenphase gefunden. Dabei nimmt die spezifische Aktivität um das 5-6fache zu. Die Tributyrinase bleibt in der wäßrigen Phase, sie reichert sich in der Zwischenphase nicht an. Bei der Verwendung von Butanol kommt es in der Enzymlösung zum Ausfall von Eiweiß mit einer völligen Zerstörung der Enzymaktivitäten. Bei Amylalkohol fällt ebenfalls Eiweiß aus, die Enzyme werden jedoch nicht vollständig inaktiviert. Bei Hexan bildet sich keine Zwischenschicht aus. Die Enzyme der wäßrigen Phase werden durch Hexan nicht inaktiviert.

\section{Diskussion}

Die prozentuale Verteilung der lipolytischen Aktivität auf die beiden Ultrazentrifugenfraktionen hängt (bei konstanten Zentrifugationsbedingungen) von den Bedingungen, unter welchen die Enzymaktivitäten bestimmt wurden, ab. Bei Bestimmung mit demselben Substrat, aber bei unterschiedlichen $\mathrm{pH}$-Werten ergaben sich verschiedene Verteilungen. Bei $\mathrm{pH} 8,0$, optimalen Bedingungen der Lipoproteid-Lipase (Substrat: aktiviertes Triolein) findet man 60\% der Aktivität im Úberstand, bei pH 6,5 dagegen nur 20\%. Bei optimalen Bedingungen der Sediment-Lipase (Substrat: Triolein + Albumin, pH 6,5) wird im Sediment $75 \%$ der Lipaseaktivität, bei pH 8,0 aber nur $60 \%$ gefunden. Die Divergenz der Ergebnisse wird umso größer sein, je besser die Lipoproteid-Lipase bzw. die Sediment-
Lipase voneinander getrennt sind, ihr sind allerdings in dem Unterschied der pH-Optima bzw. der Differenz der spezifischen Enzymaktivitäten bei den einzelnen Substraten die Asymptoten vorgegeben.

Die Lipase des Úberstandes, die wir wegen ihrer Substratspezifität als Lipoproteid-Lipase bezeichnen, scheint mit der von GORDON (15) bzw. BJöRNTORP (3) beschriebenen $\mathrm{pH}-8,5-$ Lipase identisch $\mathrm{zu}$ sein. Es besteht auch große Ähnlichkeit mit der von KoRN (2) - beschriebenen Lipoproteid-Lipase, wenn man die Substratspezifität, das pH-Optimum und die Inhibitorwirkungen vergleicht. Die Lipoproteid-Lipase kann von der ebenfalls im Überstand lokalisierten Tributyrinase getrennt werden. Die Tributyrinase bleibt bei der Zwischenphasenverteilung in der wäßrigen Phase, während $70 \%$ der Lipoproteid-Lipase-Aktivität in der Zwischenphase gefunden wird.

Die Sediment-Lipase zeigt charakteristische Merkmale der von RIZACK (4) bzw. BJöRNTORP (3) beschriebenen Adrenalinempfindlichen Lipase. Sie wird durch $\mathrm{NaF}$ gehemmt und kann durch Adrenalin aktiviert werden, wenn das Sediment 5-6 Tage eingefroren war $(5 \mu \mathrm{g}$ Adrenalin $/ \mathrm{m} l$ ).

Beide Lipasen werden durch SH-Reagenzien gehemmt. Die Integrität der SH-Gruppen scheint für die Enzymaktivität wichtig zu sein. Die Hemmung durch $p$ Chlormercuribenzoat kənn durch äquivalente Mengen Cystein verhindert bzw. aufgehoben werden. Da der Aktivitätsverlust der 5-6 Tage lang eingefrorenen Enzyme ebenfalls durch Cystein aufgehoben wird, liegt die Annahme nahe, daß dem "Alterungsprozeß" der Enzyme Veränderungen an den SH-Gruppen zugrunde liegen. Die Inaktivierung der Enzyme durch Erwärmen $\left(50^{\circ}, 10\right.$ Min.) scheint anderer Natur zu sein. Sie kann durch Cystein nicht aufgehoben werden.

Ich danke der Deutschen Forschungsgemeinschaft für Sachbeihilfen und Frl. Christa Dorow für ihre fleißige, gewissenhafte Mitarbeit.

\section{Literatur}

1. KorN, E. D., Methods biochem. Analysis 7, 145 (1959). 2. Korn, E. D., J. biol. Chemistry 215, 1 (1955). - 3. BJörntorp, P., Amer. J. Physiol. 203, 316 (1962). - 4. Rrzack, M., J. biol. Chemistry 236, 657 (1961). - 5. KorN, E. D. und T. W. QuigleY, Biochim. biophysica Acta Amsterdam 18, 143 (1955). - 6. KorN, E. D., Science Washington 120, 399 (1954). - 7. Priz, W. und

I. JohanN, Hoppe-Seylers Z. physiol. Chem. 348, 73 (1967). -

8. Hollenberg, C. H., Amer. J. Physiol. 197, 667 (1959). -

9. Porkajac, N. und W. J. Losow, Biochim. biophysica Acta.
Amsterdam 139, 123 (1967). - 10. Vaughan, M. und S. E. Berger, J. biol. Chemistry 239, 401 (1964). - 11. Dole, V. P., J. Clin. Invest. 35, 150 (1956). - 12. Lowry, O. H. und N. J. Rosenbrough, J. biol. Chemistry 193, 265 (1951). - 13. Baskys, B., E. Klein und W. F. Lever, Federation Proc. 20, 237 (1961). 14. Gordon, R. S., E. Boyle und R. K. Brown, Proc. Soc. exp. Biol. Med. 84, 168 (1953). - 15. Gordon, R. S. und A. Cherkes, Proc. Soc. exp. Biol. Med. 97, 150 (1958).

Dr. U. H. Klemens 1 Berlin 19 Spandauer Damm 130 